\title{
Development of flexible and efficient strategies for optimizing chromatographic separations ${ }^{\text {23 }}$
}

\author{
P.F. Vanbel * \\ Department of Chemical and Physico-Chemical Analysis of Drugs, Université Catholique de Louvain, UCL 72.30, Av. E. Mounier, \\ 72, B-1200, Brussels, Belgium
}

Received 15 May 1998; received in revised form 18 August 1998; accepted 25 August 1998

\begin{abstract}
The intensive research in chemometrics is resulting in continuous development of new concepts and optimization methods. The practical chromatographic optimization examples described in this paper highlight the importance of developing efficient and flexible optimization strategies, which are adapted to the (complex) separation problems encountered in the real chromatographic world. The availability of efficient and user-friendly software should contribute to a more systematic use of chemometrical approaches. Two primordial aspects are discussed in more details: (1) the selection of adequate optimization criteria and (2) the optimum robustness. (C) 1999 Elsevier Science B.V. All rights reserved.
\end{abstract}

Keywords: Chromatography; Method development; Optimization; Robustness

\section{Introduction}

Chemometrics have found widespread use to develop selectivity optimization procedures in liquid chromatography [1-5]. This approach allows the systematic development of optimal chromatographic methods. However, despite the initial enthusiasm for computer-aided HPLC methoddevelopment systems, their acceptance in routine practice is slow. One of the main reason for the

\footnotetext{
Presented at the Drug Analysis 1998 meeting, Brussels, Belgium, 11-15 May, 1998.

* Present address: Humont 21, B-7901 Thieulain, Belgium. Tel.: + 32-2-7647230; fax: + 32-2-7647363.

E-mail address: pvanbel@club-internet.fr (P.F. Vanbel)
}

modest advent of (commercial) optimization software packages is that their configuration is too simplistic to deal with the complex problems encountered in practice. Typical examples are the presence of asymmetrical peaks or peaks of very different areas (e.g. in the field of purity analysis of drugs) and the existence of irrelevant peaks which may interfere with the separation of the relevant solutes in a complex matrix (e.g. biological samples, environmental samples).

The objective of this paper is to demonstrate with some practical examples the importance of developing flexible and efficient optimization strategies. Three important aspects are considered in this work: (1) the possibility of using various (flexible) experimental designs; (2) the selection of 
different optimization criteria adapted to complex separation problems and (3) the robustness of the optimum. The two last topics are discussed more specifically.

\section{Experimental}

\subsection{Chromatographic conditions and optimization procedures}

In this paper, the described optimization examples are based on experimental data already reported in previous articles [6,7]. The first set of data [6] concerned a mixture of four acidic solutes (benzoic acid, m-nitrobenzoic acid, 3,5-dinitrobenzoic acid and salicylic acid). However, one additional solute (aspirin) is included here. A reversed-phase liquid chromatography was chosen for this study. A 5- $\mu \mathrm{m} \mathrm{C18} \mathrm{LiChrospher} \mathrm{column}$ $(125 \times 4 \mathrm{~mm}$ I.D. $)$ and a $5-\mu \mathrm{m}$ C18 LiChrospher precolumn $(4 \times 4 \mathrm{~mm}$ I.D.) from Merck (Darmstadt, Germany) were used. The flow-rate was 1.0 $\mathrm{ml} / \mathrm{min}$ and UV detection was performed at 254 nm.

A $4 \times 3$ experimental design (three levels of methanol volume fraction and four levels of $\mathrm{pH}$ ) was used to realize the simultaneous optimization of $\mathrm{pH}$ and solvent composition (Fig. 1). The volume fraction of $\mathrm{MeOH}(\varphi)$ was varied between 0.30 and 0.40 . pH was varied between 2.76 and 6.83 with a constant total ionic strength of 0.05 M.

A sigmoidal model (Eq. (1)) was used for describing capacity factors $(k)$ :

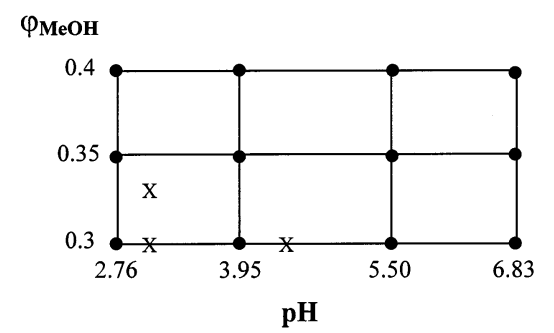

Fig. 1. The $4 \times 3$ experimental design used for the separation of a mixture of five acids. $\mathrm{X}$ refers to additional experiments. $k=$
$\frac{k_{\mathrm{HA}}^{\circ} \cdot \mathrm{e}^{S_{\mathrm{HA} \varphi+T_{\mathrm{HA}} \varphi^{2}} \cdot 10^{-\mathrm{pH}}+k_{\mathrm{A}-}^{\circ} \cdot K_{\mathrm{a}}^{\circ} \cdot \mathrm{e}^{\left(S_{\mathrm{A}-}+Q_{1}\right) \varphi+\left(T_{\mathrm{A}-}+Q_{2}\right) \varphi^{2}}}}{10^{-\mathrm{pH}}+K_{\mathrm{a}}^{\circ} \cdot \mathrm{e}^{\left(Q_{1} \varphi+Q_{2} \varphi^{2}\right)}}$

where $k_{\mathrm{HA}}^{\circ}$ and $k_{\mathrm{A}-}^{\circ}$ are extrapolated capacity factors of, respectively, the acid and the basic forms of the solute in pure water, $K_{\mathrm{a}}^{\circ}$ is the extrapolated acid-dissociation constant in pure water, $S_{\mathrm{HA}}$ and $T_{\mathrm{HA}}$ are parameters describing the variation of retention with $\varphi$ for acid species, $S_{\mathrm{A}-}$ and $T_{\mathrm{A}-}$ are corresponding parameters for basic species, and $Q_{1}$ and $Q_{2}$ are coefficients describing the variation of the acid-dissociation constant with $\varphi$.

Eq. (1) was also used to model peak heights and peak areas.

A quadratic expression (Eq. (2)) was used to model the asymmetry factors $\left(A_{\mathrm{S}}\right)$ :

$$
\begin{aligned}
A_{\mathrm{S}}= & b_{0} \cdot \mathrm{e}^{S_{0} \varphi+T_{0} \varphi^{2}}+b_{1} \cdot \mathrm{e}^{\left(S_{1} \varphi+T_{1} \varphi^{2}\right)} \cdot \mathrm{pH} \\
& +b_{2} \cdot \mathrm{e}^{\left(S_{2} \varphi+T_{2} \varphi^{2}\right)} \cdot \mathrm{pH}^{2}
\end{aligned}
$$

A detailed description of the chromatographic conditions and optimization procedure can be found in reference [6].

The second set of data concerned a mixture of nine benzodiazepines.(demoxepam, nitrazepam, oxazepam, clonazepam, flunitrazepam, triazolam, nordazepam, diazepam and ethyl loflazepate). A reversed-phase system was chosen for this study. A $5 \mu \mathrm{m} 60$ RP-Select B LiChrospher column $(125 \times 4 \mathrm{~mm}$ I.D.) and a $5 \mu \mathrm{m} 60$ RP-Select B LiChrospher precolumn $(4 \times 4 \mathrm{~mm}$ I.D. $)$ from Merck (Darmstadt, Germany) were used. The temperature of the column was maintained at $35^{\circ} \mathrm{C}$. The flow-rate was $1.0 \mathrm{ml} / \mathrm{min}$. Chromatograms were recorded at $230 \mathrm{~nm}$. A conventional procedure was used for optimizing the volume fraction of several organic modifiers in the mobile phase, i.e. ACN, MeOH and THF. The experimental design is shown in Fig. 2. An exponentially quadratic equation (Eq. (3)) was used to model the capacity factor $(k)$ as a function of the mobile phase composition:

$k=a_{1} \cdot \mathrm{e}^{a_{2} \cdot \bar{\varphi}_{1}+a_{3} \cdot \bar{\varphi}_{2}+a_{4} \cdot \bar{\varphi}^{2}+a_{5} \cdot \bar{\varphi}_{2}^{2}+a_{6} \cdot \bar{\varphi}_{1} \cdot \bar{\varphi}_{2}}$ 


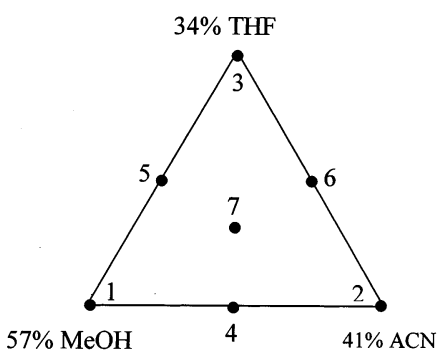

Fig. 2. Experimental design used for the separation of a limited number of solutes from a mixture of nine benzodiazepines.

where $\bar{\varphi}_{1}$ is the volume fraction of one solvent (i.e. the binary mixture $\mathrm{ACN} / \mathrm{H}_{2} \mathrm{O}$ ) in the mobile phase and $\varphi_{2}$ is the volume fraction of a second solvent (i.e. the binary mixture $\mathrm{MeOH} / \mathrm{H}_{2} \mathrm{O}$ ).

\subsection{Software}

Different 'in house' software programs were developed to model capacity factors and other chromatographic parameters (peak heights, peak areas and asymmetry factors) and to generate response surfaces. Software is also available for generating predicted or simulated chromatograms. All the programs were written in Pascal (Turbo Pascal 7.0, Borland International, Scotts Valley, CA, USA) and implemented on an IBM-compatible computer. Data generated by Pascal programs were imported directly in Excel software (version 5.0) in a Windows environment (Microsoft Corporation) for further manipulation and graphical presentation.

\section{Results and discussion}

\subsection{Optimization criteria}

Many different criteria have been suggested in the literature to assess the quality of chromatographic separations [1,2,7-9]. Optimization criteria that adequately describe the quality of the separation are among the essential factors determining the applicability of selectivity-optimization procedures. Indeed, the result of an optimization process depends on the selected optimization criterion, so that the latter has to be defined in the context of the objectives of the separation. Fig. 3 presents three simulated chromatograms including one huge asymmetrical peak and three small peaks. A $t_{0}$ peak is considered at $t=1.5 \mathrm{~min}$. The chromatographic parameters (retention times, peak heights, number of theoretical plates and asymmetry factors) are given in Table 1.

The minimum resolution (lowest value of resolution selected from the relevant pairs of peaks) is the most classical overall optimization criterion. Eq. (4) is largely used to calculate the chromatographic resolution $\left(R_{\mathrm{S}}\right)$ :

$R_{\mathrm{S}, j i}=\frac{\left(k_{j}-k_{i}\right)}{\left(k_{j}+k_{i}+2\right)} \frac{\sqrt{\bar{N}}}{2}$

where $k$ is the capacity factor and $\bar{N}$ is the average number of theoretical plates.

Resolution can also be calculated by using more complex expressions as the effective resolution, $R_{1}$ (Eqs. (5a) and (5b)):

$$
\begin{aligned}
& \frac{{ }^{i} R_{n}=}{\frac{\left(t_{R, j}-t_{R, i}\right)\left(1+A_{S, i}\right)\left(1+A_{S, j}\right) \sqrt{N_{i} N_{j}}}{4 A_{S, i} t_{R, i}\left(1+A_{S, j}\right) \sqrt{N_{j}}+4 t_{R, j}\left(1+A_{S, i}\right) \sqrt{N_{i}} \sqrt{1+0.5 \ln \left(h_{j} / h_{i}\right)}}} \\
& \frac{(5 \mathrm{a})}{{ }^{j} R_{p}=\quad} \\
& \frac{\left(t_{R, j}-t_{R, i}\right)\left(1+A_{S, i}\right)\left(1+A_{S, j}\right) \sqrt{N_{i} N_{j}}}{4 A_{S, i} t_{R, i}\left(1+A_{S, j}\right) \sqrt{N_{j}} \sqrt{1+0.5 \ln \left(h_{i} / h_{j}\right)}+4 t_{R, j}\left(1+A_{S, i}\right) \sqrt{N_{i}}}
\end{aligned}
$$
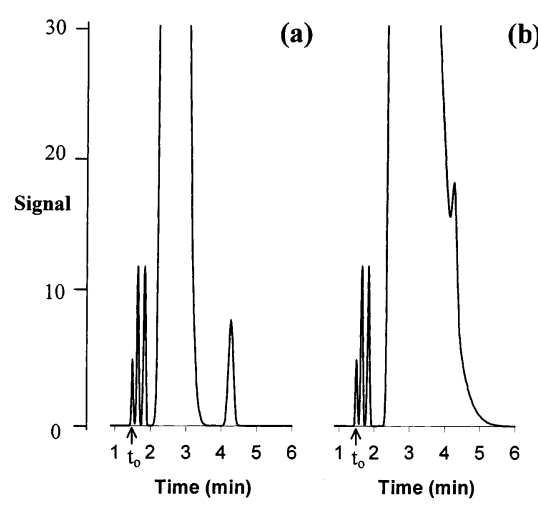

(b)

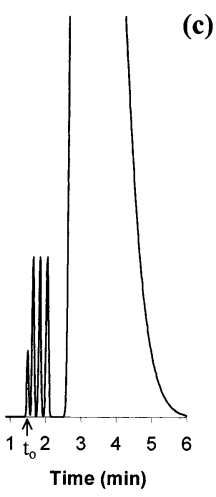

Fig. 3. Computer-simulated chromatograms. The chromatographic parameters are given in Table 1. 
Table 1

Chromatographic parameters (retention time, $t_{\mathrm{R}}$; peak height, $h$; number of theoretical plates, $N$ and, asymmetry factor, $A_{S}$ ) and values of resolution $\left(R_{S}\right)$ and effective resolution $\left(R_{l}\right)$ corresponding to the computer-simulated chromatograms presented in Fig. 3a-c

Chromatogram presented in Fig. $3 \mathrm{a}=$ chromatogram a

\begin{tabular}{llrrrrr}
\hline Peak & $t_{\mathrm{R}}(\min )$ & \multicolumn{1}{l}{$h$} & $N$ & $A_{S}$ & $R_{S}$ & $R_{l}$ \\
\hline $0^{*}$ & 1.50 & 5 & 2000 & 1 & - & - \\
1 & 1.65 & 12 & 2000 & 1 & - & - \\
2 & 1.84 & 12 & 2000 & 1 & 1.2 & 1.2 \\
3 & 2.45 & 700 & 100 & 3 & 2.3 & 1.2 \\
4 & 4.27 & 8 & 2000 & 1 & 4.4 & 1.2
\end{tabular}

Chromatogram presented in Fig. $3 b=$ chromatogram $b$

$\begin{array}{llrrrrl}0^{*} & 1.50 & 5 & 2000 & 1 & - & - \\ 1 & 1.65 & 12 & 2000 & 1 & - & - \\ 2 & 1.84 & 12 & 2000 & 1 & 1.2 & 1.2 \\ 3 & 2.45 & 140 & 17 & 17 & 2.3 & 2.2 \\ 4 & 4.27 & 8 & 2000 & 1 & 4.3 & 0.5\end{array}$

Chromatogram presented in Fig. $3 c=$ chromatogram $c$

$\begin{array}{llrrrrl}0^{*} & 1.50 & 5 & 2000 & 1 & - & - \\ 1 & 1.65 & 12 & 2000 & 1 & - & - \\ 2 & 1.84 & 12 & 2000 & 1 & 1.2 & 1.2 \\ 3 & 2.05 & 12 & 2000 & 1 & 1.2 & 1.2 \\ 4 & 2.73 & 130 & 17 & 17 & 2.3 & 2.2\end{array}$

* Peak 0 corresponds to the column hold-up time.

where $t_{R}$ is the retention time, $A_{S}$ the asymmetry factor, $N$ the number of theoretical plates and $h$ the peak height.

This resolution function developed by Schoenmakers et al. [10] is especially applicable in nonideal situations (chromatograms containing asymmetrical peaks and/or peaks of vastly different areas). It takes into account the individual widths of the two peaks, the asymmetry factors, and the peak heights. When the separation of a pair of peaks is considered, two values of the effective resolution exist. The first one, ${ }^{i} R_{n}$, describes the extent to which peak $i$ is separated from the next peak $(j)$ and the second value, ${ }^{j} R_{p}$, reflects the extent to which peak $j$ is separated from the previous one (i). Generally, in complete optimization (all peaks are relevant), the lowest value characterizing a particular separation (i.e. ${ }^{i} R_{n}$ or ${ }^{j} R_{p}$ ) is kept.

For each chromatogram presented in Fig. 3, Table 2 lists the values of the minimum resolu- tion, $R_{S}$, min, calculated with the classical formula (Eq. (4)) and of the minimum effective resolution, $R_{l, \text { min }}$ (Eqs. (5a) and (5b)). The minimum resolution does not differentiate between chromatograms $a$ and $b$ although the resolution of the last two peaks is much better in chromatogram a than in chromatogram b. Indeed, chromatograms $a$ and $b$ show the same retention times, but the huge peak in chromatogram $b$ is wider and more asymmetrical than in chromatogram a. This demonstrates the fundamental importance of selecting an appropriate optimization criterion. The classical resolution expression (Eq. (4)) does not describe adequately the quality of the separation between the huge asymmetrical peak and the small adjacent peak. In non-ideal situations the effective resolution is the recommended choice. In this example, the effective resolution correctly describes the separation between the huge asymmetrical peak and its neighbors (Table 1).

The calculation of some criteria requires the estimation of the analysis time. For example, the objective of threshold resolution is to minimize the analysis time when a certain value of resolution is reached (e.g. 1.5). Generally, the retention time of the last peak is an adequate approximation of the analysis time (chromatogram a). However, this is no longer appropriate when a huge asymmetrical peak appears at the end of the chromatogram (Fig. 3b-c). In Fig. 3c, for example, the retention time of the last peak is equal to $2.73 \mathrm{~min}$ but the analysis time is longer than 6 min. In such case, the analysis time has to be calculated by using the following equation which

Table 2

Values of analysis time $\left(t_{\text {analysis }}\right)$, minimum resolution $\left(R_{S}\right.$, $\min )$, and of the minimum effective resolution $\left(R_{l, \min }\right)$ corresponding to the chromatograms presented in Fig. $3 \mathrm{a}-\mathrm{c}$

\begin{tabular}{llll}
\hline & \multicolumn{3}{l}{ Chromatogram } \\
\cline { 2 - 4 } & $\mathrm{a}$ & $\mathrm{b}$ & $\mathrm{c}$ \\
\hline$R_{S, \text { min }}$ & 1.2 & 1.2 & 1.2 \\
$R_{l, \text { min }}$ & 1.2 & 0.5 & 1.2 \\
$t_{\mathrm{R}}$ (last peak) (min) & 4.27 & 4.27 & 2.73 \\
$t_{\text {analysis }}$ (min) & 4.46 & 5.95 & 6.43 \\
\hline
\end{tabular}


takes into account peak asymmetry and the difference in peak heights $[7,10]$ :

$t_{\text {analysis }}=t_{R}\left\{1+\frac{4 A_{S}}{\sqrt{N}\left(1+A_{S}\right)} \sqrt{1+0.5 \ln \left(h_{\mathrm{hp}} / h_{\mathrm{sp}}\right)}\right\}$

where $h_{\mathrm{hp}}$ is the height of the huge peak and $h_{\mathrm{sp}}$ is the height of the small adjacent peak.

The selection of an adequate criterion is a important step for the success of any optimization procedure. Another fundamental aspect is the flexibility of optimization criteria. For example, analysts are often interested in the separation of a limited number of solutes in a complex mixture (e.g. biological and environmental samples). Limited optimization refers to this kind of situation. In order to optimize the separation of five acidic solutes (benzoic acid, m-nitrobenzoic acid, 3,5dinitrobenzoic acid, salicylic acid and aspirin), the minimum effective resolution is selected as optimization criterion. The optimized parameters are the $\mathrm{pH}$ of the mobile phase and the percentage of $\mathrm{MeOH}$ in the mobile phase (see experimental design in Fig. 1). A complete description of the experimental data can be found in ref. [6]. When all five solutes are relevant, the chromatogram presented in Fig. 4a is selected as optimum by the minimum effective resolution $\left(R_{l, \min }=1.2\right)$. However, if the chromatographer is only interested in the separation of two solutes among the five (i.e. benzoic acid and dinitrobenzoic acid), another chromatogram is the optimum (Fig. 4b). The minimum effective resolution is selected from the relevant pairs of peaks. Irrelevant peaks do not need to be separated from each other. Compared to the complete optimization process (Fig. 4a), the value of $R_{1, \text { min }}$ is higher (2.95) and the analysis time is about $6 \mathrm{~min}$ instead of $12 \mathrm{~min}$. Fig. $4 \mathrm{c}$ is the optimum chromatogram when dinitrobenzoic is the only interesting peak. This example is particularly favorable. $R_{l, \min }$ is equal to 5.1 and the analysis time is lower than $5 \mathrm{~min}$. This is due to the fact that irrelevant peaks appear at the beginning of the chromatogram and are almost all confounded. These examples illustrate clearly the interest of considering the relevance of the peaks during an optimization process. (a)

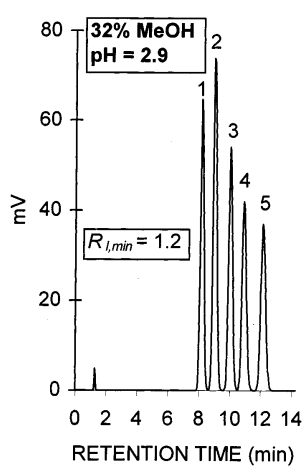

(b)

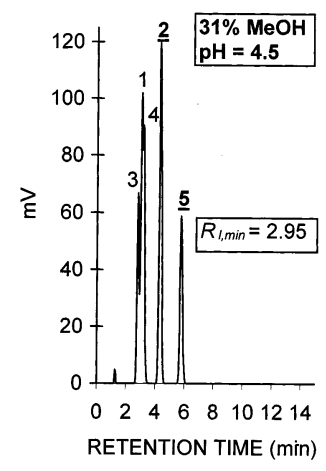

(c)

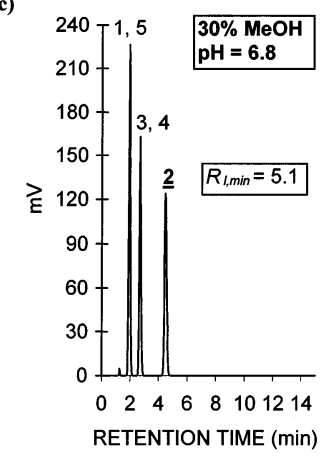

Fig. 4. Optimum chromatograms defined by the minimum effective resolution when (a) all five solutes are relevant; (b) benzoic acid and dinitrobenzoic acid are relevant; and (c) only dinitrobenzoic acid is relevant. Peaks: $1=$ aspirin; $2=3,5$-dinitrobenzoic acid; 3 = salicylic acid; $4=$ m-nitrobenzoic acid and $5=$ benzoic acid. In chromatograms $\mathrm{b}$ and $\mathrm{c}$, relevant peak numbers are underlined.

Other specific objectives can be achieved by using particular optimization criteria. So, the calibrated normalized resolution product, $\underline{r}^{*}$ (Eq. (7)) promotes situations where relevant peaks are equally distributed over the chromatogram (same resolution) and where, ideally, all irrelevant peaks are confounded with a (hypothetical) $t_{0}$ peak [7]. When these two conditions are fulfilled, $\underline{r}^{*}$ is equal to one. Although this ideal situation is not often met in practice, an important practical consequence of using this type of criteria is that it favors separations where the relevant peaks are adjacent and where irrelevant peaks are confounded.

$\underline{r}^{*}=\prod_{k=1}^{d}\left(\underline{c}_{k} / \bar{c}\right)$ 
with

$$
\bar{c}=\frac{1^{n}}{p_{i=0}-1} \sum_{j i}
$$

where $c$ is the elementary criterion $\left(R_{S}, R_{l} \ldots\right), n$ the number of solutes, $\underline{c}_{k}$ the relevant values of $c$, and $p$ the number of relevant peaks.

The following application illustrates the characteristics of the calibrated normalized resolution product. The optimization of the mobile phase composition (volume fractions of $\mathrm{MeOH}, \mathrm{ACN}$ and THF) for a mixture of nine benzodiazepines is achieved using a classical mixture design (Fig. 2). Experimental details can be found in ref. [7]. Fig. 5a is the optimum chromatogram selected by $\underline{r}^{*}$ when nordazepam, diazepam and ethyl loflazepate are relevant. The interesting peaks are adjacent while irrelevant peaks tend to be confounded (four irrelevant peaks are coeluting: triazolam, oxazepam, flunitrazepam and clonazepam). Fig. 5b shows another example of limited optimization where flunitrazepam, triazolam
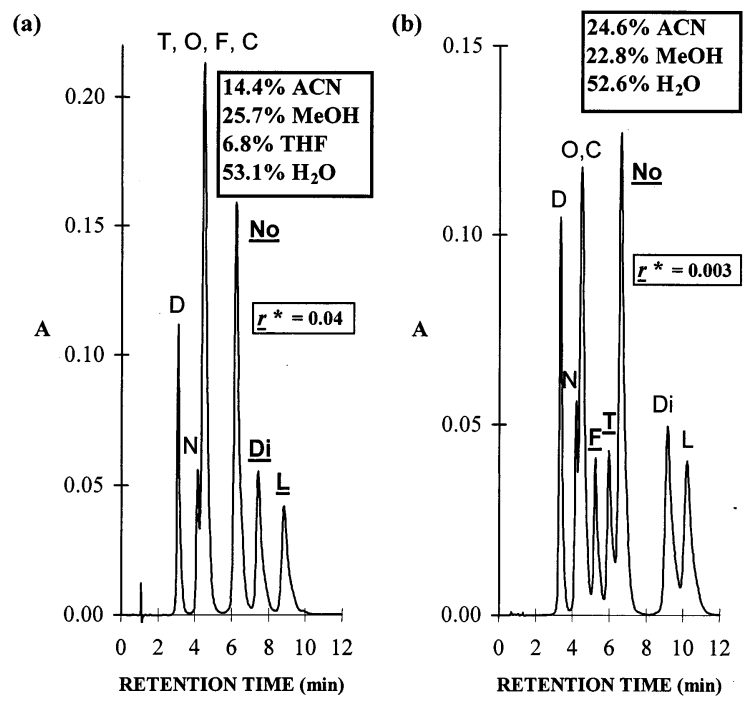

Fig. 5. Optimum chromatograms selected by the calibrated normalized resolution product based on the separation factor when (a) nordazepam, diazepam and ethyl loflazepate are the relevant solutes; and (b) flunitrazepam, triazolam and nordazepam are relevant. Peaks: $\mathrm{T}=$ triazolam; $\mathrm{D}=$ demoxepam; $\mathrm{O}=$ oxazepam; $\mathrm{F}=$ flunitrazepam; No = nordazepam; $\mathrm{N}=$ nitrazepam; $\mathrm{C}=$ clonazepam; $\mathrm{Di}=$ diazepam and $\mathrm{L}=$ ethyl loflazepate. Relevant peak numbers are underlined. (a)

(b)
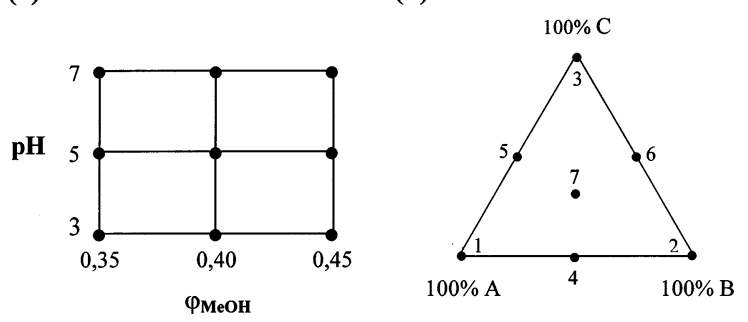

Fig. 6. Classical experimental designs: examples of (a) factorial design; and (b) mixture design.

and nordazepam are relevant. The peaks of interest are again adjacent in this optimum chromatogram. In comparison with the chromatogram presented in Fig. 5a, where triazolam and flunitrazepam are irrelevant and confounded with two other irrelevant peaks, these two compounds are now separated from the irrelevant peaks. $\underline{r}^{*}$ promote the separation of solutes into groups. These examples again demonstrate the importance of considering the relevance of the peaks during an optimization strategy.

In conclusion, improved optimization criteria which can deal with non-ideal peaks and with limited optimization now exist [7] and these should be integrated in all optimization software.

\subsection{Experimental designs and mathematical modelling}

Another requirement is the possibility to select appropriate experimental designs and mathematical models to optimize different parameters. Classical experimental designs (e.g. mixture designs or classical factorial designs) are generally available in optimization software (Fig. 6). However, more complex situations ( $\mathrm{pH}$ optimization) can require more complex mathematical modelling and the use of irregular designs [11-15] or D-optimal designs [16].

\subsection{Robustness of the optimum}

The robustness of the optimum is also a very important aspect. The US Pharmacopeia [17] defines robustness as: "the robustness of an analytical procedure is a measure of its capacity to remain 
unaffected by small but deliberate variations in method parameters and provides an indication of its reliability during normal usage". The robustness of a method is typically evaluated at the final stage of method development (validation process). However, it is of great potential benefit to consider the robustness of chromatographic separations at an early stage of method development. This greatly reduces the risk of major disappointments when seemingly good methods fail a ruggedness test. To illustrate the concept of robustness, a fictive response surface of resolution with respect to $\mathrm{pH}$ is presented in Fig. 7. Strictly, the optimum value of resolution (maximum value) is located at $\mathrm{pH} 3$. However, the response surface is very sharp in this region and this optimum is not robust at all. Small variation of $\mathrm{pH}$ leads to dramatic changes in terms of resolution. The selection of another optimum around $\mathrm{pH} 4.5$ is far more appropriate in this case. Resolution is good and the response surface presents a plateau in this $\mathrm{pH}$ range. Note that another robust region is located around $\mathrm{pH} 6.5$ but the resolution is close to zero. Robustness can not be a goal in itself in method development procedure and it must be combined with other quality criteria (resolution, analysis time...).

Robustness can be included as an objective in optimization strategies by using robustness criteria. In previous work, Vanbel et al. [6] defined such criteria as derivatives of the minimum resolution with respect to the optimized parameters (Eqs. (9) and (10)).

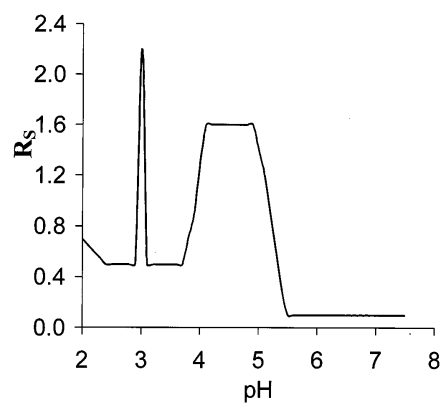

Fig. 7. Fictive response surface (resolution with respect to $\mathrm{pH}$ ) to illustrate the concept of robustness.

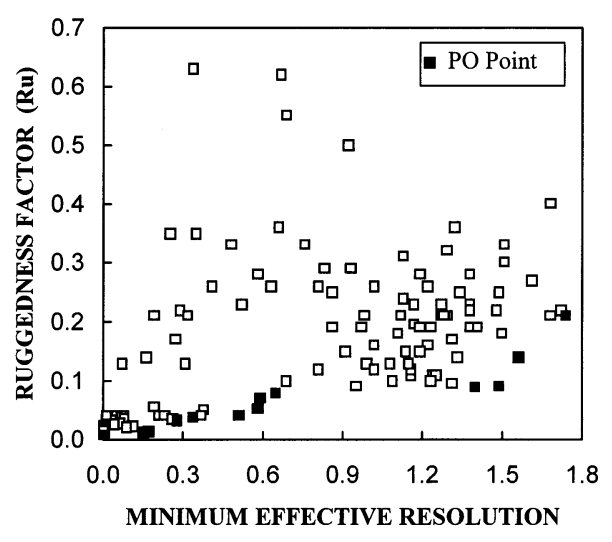

Fig. 8. Example of Pareto-optimality plot obtained during the optimization of the separation of four acidic solutes (optimization criteria: minimum effective resolution and robustness criterion, $R_{u}$ ).

$R_{u}=\sum_{i=1}^{n}\left(\Delta_{x_{i}} \frac{\mathrm{d} R_{S, \min }}{\mathrm{d} x_{i}}\right)$

$\left[R_{u}^{*}\right]^{-1}=\frac{R_{S, \min }}{R_{u}}$

where $R_{S, \text { min }}$ is the minimum resolution, $x_{i}$ the optimized parameter $i$, and $\Delta x_{i}$ the permitted variation of parameter $x_{i}$.

The implementation of multi criteria decision making (MCDM) techniques is required to find a suitable compromise between robustness and chromatographic resolution (and/or other objectives such as the analysis time). Fig. 8 shows an example of a Pareto-optimality plot for the minimum effective resolution and the robustness criterion, $R_{u}$, obtained during the optimization of $\mathrm{pH}$ and mobile composition for the separation of four acidic solutes [6]. Resolution has to be maximized and $R_{u}$ has to be minimized. The MCDM plot visualizes directly the pay-off between the two criteria. Information with respect to both criteria is available, so that the chromatographer can decide which of the Pareto-optimal (PO) points is preferable.

In a recent study, Massart [18] suggest the use of optimization functions which are multicriteria in nature. Quality of the separation and robustness are combined in the same mathematical equation (Eqs. (11) and (12)). 


$$
\begin{aligned}
& C R_{1}=n\left(\frac{\left(f_{j}\right)_{S}}{\sum_{i=1}^{n}\left|\frac{\Delta\left(f_{j i}\right)_{S}}{\Delta x}\right|}\right) \\
& C R_{2}=\left(\frac{\left(f_{j}\right)_{S}}{\prod_{i=1}^{n}\left(1+\left|\frac{\Delta\left(f_{j i}\right)_{S}}{\Delta x}\right|\right)}\right)
\end{aligned}
$$

where $\left(f_{j}\right)_{\mathrm{S}}$ is the scaled response for a point $j$ and $\Delta x$ the variation of parameter $x$.

This approach does not need the use of MCDM techniques to find an appropriate optimum. However, a single number describes the overall quality of the separation and the chromatographer sacrifices control of the optimization process.

Considering robustness as an objective from the beginning of method development reduces significantly the chance of failure during the validation process. This concept should be systematically integrated in any optimization procedure. Method validation is still a recommended step.

\section{Conclusion}

Developing flexible and efficient optimization strategies is required to encounter the various objectives of chromatographers. Optimization software should not be real black boxes and should provide:

1. adequate and flexible optimization criteria adapted to practical situations (presence of non ideal peaks, existence of irrelevant interfering peaks...);

2. various (flexible) experimental designs and modelling equations;

3. an indication of optimum robustness.

The availability of such flexible optimization strategies and tools should contribute to a more systematic use of computer-aided HPLC method development systems.

\section{Acknowledgements}

The author thanks Professor B. Tilquin (Université Catholique de Louvain, Belgium), Dr B. Rollmann (Université Catholique de Louvain, Belgium) and Dr P.J. Schoenmakers (Shell Research, Amsterdam, The Netherlands) for fruitful discussions and comments.

\section{References}

[1] J.C. Berridge, Techniques for the Automated Optimization of HPLC Separations, Wiley, Chichester, 1985.

[2] P.J. Schoenmakers, Optimization of Chromatographic Selectivity. A Guide to Method Development, Elsevier, Amsterdam, 1986.

[3] P.J. Schoenmakers, M. Mulholland, Chromatographia 25 (1988) 737-748.

[4] P.J. Schoenmakers, A. Peeters, R.J. Lynch, J. Chromatogr. 506 (1990) 169-184.

[5] L.R. Snyder, J.L. Glajch, J.J. Kirkland, Practical HPLC Method Development, Wiley, New York, 1988.

[6] P.F. Vanbel, B.L. Tilquin, P.J. Schoenmakers, J. Chromatogr. 697 (1995) 3-16.

[7] P.F. Vanbel, B.L. Tilquin, P.J. Schoenmakers, Chemom. Intell. Lab. Syst. 35 (1996) 67-86.

[8] S. Hatrik, J. Hrouzek, Chem. Listy 89 (1995) 410-417.

[9] P.F. Vanbel, Optimization criteria in high-performance liquid chromatography, Ph.D. thesis, Université catholique de Louvain, Bruxelles, 1997.

[10] P.J. Schoenmakers, J.K. Strasters, A. Bartha, J. Chromatogr. 458 (1988) 355-370.

[11] R.M. Lopes Marques, P.J. Schoenmakers, J. Chromatogr. 592 (1992) 157-182.

[12] P.J. Schoenmakers, R. Tijssen, J. Chromatogr. 656 (1993) 577-590.

[13] B. Bourguignon, P.F. de Aguiar, M.S. Khots, D.L. Massart, Anal. Chem. 66 (1994) 893-904.

[14] B. Bourguignon, P.F. de Aguiar, K. Thorre, D.L. Massart, J. Chromatogr. Sci. 32 (1994) 144-152.

[15] S.M. Murray, Advances in HPLC method-development relevant to the pharmaceutical industry, Ph.D. thesis, University of Strathclyde, Glasgow, 1996, Ch. 4.

[16] P.F. de Aguiar, B. Bourguignon, M.S. Khots, D.L. Massart, R. Phan-Tan-Luu, Chemom. Intell. Lab. Syst. 30 (1995) 199-210.

[17] The United States Pharmacopeia 23, The National Formulary 18, United States Pharmacopeial Convention, Rockville, 1995, p. 1983.

[18] P.F. de Aguiar, Y. Vander Heyden, D.L. Massart, Anal. Chim. Acta 348 (1997) 223-235. 